\title{
Author Correction: Paternal grandfather's access to food predicts all-cause and cancer mortality in grandsons
}

\author{
Denny Vågerö (D), Pia R. Pinger (D, Vanda Aronsson (D) \& Gerard J. van den Berg (D)
}

Correction to: Nature Communications https://doi.org/10.1038/s41467-018-07617-9, published online 11 December 2018

The original version of the Supplementary information associated with this Article contained an error in Supplementary Table 7 in which the power calculations were incorrect as a result of an error in the standard deviation of the exposure variable when performing the calculations. The correct version of Supplementary Table 7 is:

Supplementary Table 7: What is the power in our replication to detect the most important results in the Överkalix studies?

\begin{tabular}{|c|c|c|c|c|c|c|}
\hline \multicolumn{7}{|c|}{$\begin{array}{l}\text { All-cause mortality result (Kaati et al., 2007) } \\
\text { Males }\end{array}$} \\
\hline Food access & Exposed ancestor & Hazard Ratio & $p$ & Deaths_Överkalix & Deaths/Total N_replication & POWER \\
\hline good & father & 1.70 & 0.01 & $146--$ & $3419 / 3820$ & $>0.99$ \\
\hline good & paternal grandfather & 1.45 & 0.05 & 164 & $339 / 3224$ & 0.48 \\
\hline poor & paternal grandfather & 0.60 & 0.01 & 164 & $339 / 3224$ & 0.62 \\
\hline \multicolumn{7}{|l|}{ Females } \\
\hline Food access & Exposed ancestor & Hazard Ratio & $p$ & Deaths_Överkalix & Deaths/Total N, replication & POWER \\
\hline good & paternal grandmother & 1.75 & 0.01 & 139 & $222 / 3051$ & 0.50 \\
\hline poor & paternal grandmother & 0.71 & 0.01 & 135 & $222 / 3051$ & 0.30 \\
\hline \multicolumn{7}{|c|}{ Diabetes and cardiovascular mortality results (Kaati et al., 2002) } \\
\hline \multicolumn{7}{|c|}{ Diabetes, males and females combined } \\
\hline Food access & Exposed ancestor & Odds Ratio & $p$ & Deaths/Total N_Överkalix & Deaths/Total $N_{-}$replication & POWER \\
\hline good & father & 0.14 & 0.06 & $19 / 239$ & $544 / 7280$ & $>0.99$ \\
\hline good & paternal grandfather & 2.34 & 0.09 & $19 / 239$ & $26 / 6275$ & 0.49 \\
\hline poor & paternal grandfather & 0.35 & 0.09 & $19 / 239$ & $26 / 6275$ & 0.06 \\
\hline poor & maternal grandmother & 2.73 & 0.06 & $19 / 239$ & $41 / 5891$ & 0.54 \\
\hline \multicolumn{7}{|c|}{ CVD, males and females combined } \\
\hline Food access & Exposed ancestor & Odds Ratio & $p$ & Deaths/Total N_Överkalix & Deaths/Total $N_{-}$replication & POWER \\
\hline poor & father & 0.42 & 0.05 & $128 / 239$ & $3846 / 7280-1$ & $>0.99$ \\
\hline
\end{tabular}

Notes: Hazard ratios and odds ratios as reported in Kaati et al. (2002) and Kaati et al. (2007). The power analyses for all-cause mortality (one-sided test) were computed using Schoenfeld's sample size-formula for the proportional hazards regression model. For Diabetes and CVD mortality the power was computed using a two-sample proportions test (one-sided test, only decreased individuals in $\mathrm{UBCoS}$ were used to determine the sample size). 
which replaces the previous incorrect version:

Supplementary Table 7: What is the power in our replication to detect the most important results in the Överkalix studies?

\begin{tabular}{|c|c|c|c|c|c|c|}
\hline \multicolumn{7}{|c|}{ All-cause mortality results (Kaati et al., 2007) } \\
\hline \multicolumn{7}{|c|}{ Males } \\
\hline Food access & Exposed ancestor & Hazard Ratio & $p$ & Deaths_Överkalix & Deaths/Total N_replication & POWER \\
\hline good & father & 1.70 & 0.01 & $146-$ & $3419 / 3820-1$ & $>0.99$ \\
\hline good & paternal grandfather & 1.45 & 0.05 & 164 & $339 / 3224$ & 0.93 \\
\hline poor & paternal grandfather & 0.60 & 0.01 & 164 & $339 / 3224$ & 0.99 \\
\hline \multicolumn{7}{|l|}{ Females } \\
\hline Food access & Exposed ancestor & Hazard Ratio & $p$ & Deaths_Överkalix & Deaths/Total N, replication & POWER \\
\hline good & paternal grandmother & 1.75 & 0.01 & 139 & $222 / 3051$ & 0.99 \\
\hline poor & paternal grandmother & 0.71 & 0.01 & 135 & $222 / 3051$ & 0.72 \\
\hline \multicolumn{7}{|c|}{ Diabetes and cardiovascular mortality results (Kaati et al., 2002) } \\
\hline \multicolumn{7}{|c|}{ Diabetes, males and females combined } \\
\hline Food access & Exposed ancestor & Odds Ratio & $p$ & Deaths/Total N_Överkalix & Deaths/Total $N_{-}$replication & POWER \\
\hline good & father & 0.14 & 0.06 & $19 / 239$ & $544 / 7280$ & $>0.99$ \\
\hline good & paternal grandfather & 2.34 & 0.09 & $19 / 239$ & $26 / 6275$ & 0.76 \\
\hline poor & paternal grandfather & 0.35 & 0.09 & $19 / 239$ & $26 / 6275$ & 0.87 \\
\hline poor & maternal grandmother & 2.73 & 0.06 & $19 / 239$ & $41 / 5891$ & 0.90 \\
\hline \multicolumn{7}{|c|}{ CVD, males and females combined } \\
\hline Food access & Exposed ancestor & Odds Ratio & $p$ & Deaths/Total N_Överkalix & Deaths/Total $N_{-}$replication & POWER \\
\hline poor & father & 0.42 & 0.05 & $128 / 239$ & $3846 / 7280$ & $>0.99$ \\
\hline
\end{tabular}

Notes: Relative probabilities were reconstructed from sample sizes and odds ratios reported in (Kaati et al., 2002). The power analyses for all-cause mortality were computed using Schoenfeld's sample-size formula for the proportional-hazards regression model. For Diabetes and CVD mortality the power was computed using a two-sample proportions test (only deceased individuals in UBCoS were used to determine the sample size).

Furthermore, the original version of this Article contained an error in the second paragraph of the 'Results' section, which incorrectly read 'In analyses of G2 mortality this power varied from 72 to $99 \%$.' The correct version reads 'In analyses of G2 mortality this power was modest'. These have been corrected in both the PDF and HTML versions of the Article and Supplementary information file. The HTML has also been updated to include a corrected version of the Supplementary information.

Published online: 23 March 2021

\section{Additional information}

Supplementary information The online version contains supplementary material available at https://doi.org/10.1038/s41467-021-22367-x.

\footnotetext{
(c) (i) Open Access This article is licensed under a Creative Commons Attribution 4.0 International License, which permits use, sharing, adaptation, distribution and reproduction in any medium or format, as long as you give appropriate credit to the original author(s) and the source, provide a link to the Creative Commons license, and indicate if changes were made. The images or other third party material in this article are included in the article's Creative Commons license, unless indicated otherwise in a credit line to the material. If material is not included in the article's Creative Commons license and your intended use is not permitted by statutory regulation or exceeds the permitted use, you will need to obtain permission directly from the copyright holder. To view a copy of this license, visit http://creativecommons.org/licenses/by/4.0/.
}

(c) The Author(s) 2021 